\title{
An evaluation of $E$. coli in urinary tract infection in emergency department at KAMC in Riyadh, Saudi Arabia: retrospective study
}

\author{
Menyfah Q. Alanazi ${ }^{*}$, Fulwah Y. Alqahtani ${ }^{2}$ and Fadilah S. Aleanizy ${ }^{2}$
}

\begin{abstract}
Background: Urinary tract infection (UTIS) is a common infectious disease in which level of antimicrobial resistance are alarming worldwide. Therefore, this study aims to describe the prevalence and the resistance pattern of the main bacteria responsible for UTIS Escherichia coli (E. coli).

Methods: Retrospective chart review for patients admitted to emergency department and diagnosed with UTIS at KAMC, in Riyadh, Saudi Arabia between January to March 2008 was performed. Antimicrobial susceptibility to ampicillin, augmentin (amoxicillin/clavulanate), cefazolin, co-trimoxazole (sulfamethoxazole/trimethoprim), ciprofloxacin, and nitrofurantoin, and cefpodoxime was determined for 101 E. coli urinary isolates.
\end{abstract}

Results: Escherichia coli was the most prevalent pathogen contributing to UTIS representing 93.55, 60.24, and 45.83\% of all pathogen isolated from urine culture of pediatric, adult, and elderly, respectively. High rates of resistance to ampicillin (82.76, 58, and 63.64\%) and co-trimoxazole (51.72, 42, and 59.09\%), among E. coli isolated from pediatric, adult and elderly respectively. Nitrofurantoin was the most active agent, followed by ciprofloxacin, augmentin and cefazolin. 22.77\% of E. coli isolates exhibited multiple drug resistance (MDR). Among 66 and 49 isolates resistant to ampicillin and co-trimoxazole, respectively, 34.84 and $42.85 \%$ were MDR. In contrast, all isolates resistant to augmentin and nitrofurantoin were MRD, while 72.7 and $82.4 \%$ of isolates resistant to ciprofloxacin and cefazolin were MDR.

Conclusions: High resistance was observed to ampicillin and co-trimoxazole which commonly used as empirical treatments for UTIS, limiting their clinical use. This necessitates continuous surveillance for resistance pattern of uropathogens against antibiotics.

Keywords: Antibiotics resistance, E. coli, Empirical therapy, UTIS

\section{Background}

Urinary tract infections (UTIs) are among the most common types of bacterial infections occurring both in the community and hospital settings $[1,2]$. There are two types of UTIs: hospital associated urinary tract infection (HAUTIs), and community-associated urinary tract infection (CAUTIs) [3]. Women are the predominant group of patients with CAUTIs [1,2]. UTIs was estimated to represent 100,000 hospitalizations, 7 million

\footnotetext{
*Correspondence: anizim1@ngha.med.sa; Dr.Monifah@gmail.com ${ }^{1}$ Clinical Pharmacy Practice, Drug Policy and Economics Center, King Abdulaziz Medical City, Ministry of National Guard, Health Affairs, Riyadh 22490, Saudi Arabia

Full list of author information is available at the end of the article
}

visits and 1 million admissions to emergency services in USA $[4,5]$. The economic and public health burdens of UTIs is substantial and markedly affect the quality of life of infected patients [6]. In Saudi Arabia, UTIs were reported to be the second leading cause of infections predominately in women at ED [7].

The majority of UTIs are caused by E. coli bacteria, followed by Proteus spp., Staphylococcus saprophyticus, Klebsiella spp. and other Enterobacteriaceae [8, 9]. However, among bacteria causing UTIS, E. coli is considered as the most predominant cause of both community and nosocomial UTIs. Antibiotics commonly recommended for treatment of UTIs include co-trimoxazole (trimethoprim/sulfamethoxazole), nitrofurantoin, ciprofloxacin 
and ampicillin $[3,10]$. However, there is global increase in antibiotic resistance among urinary tract pathogens, including resistance pattern observed in Saudi Arabia [11-14], which limit treatment options.

Evidence suggest significant relationship between extensive use of antimicrobial and antimicrobial resistance $[10,11]$. Therefore, appropriate antibiotic prescription and usage will reduce the disease burden of UTIs and hence lower its complications and costs $[10,15]$. For this reason, surveillance of antibiotics resistance is crucial to determine the pattern of antimicrobial resistance and consequently guide the selection of empirical therapy. Therefore, this study aims to determine the current prevalence and susceptibility to ampicillin, augmentin (amoxicillin/clavulanate), co-trimoxazole (trimethoprim/ sulfamethoxazole), nitrofurantoin, cefazolin, and ciprofloxacin amongst all $E$. coli isolated from patients with UTIs. Associations between patient's demographic parameters and multidrug resistance $E$. coli isolates were also investigated.

\section{Methods}

\section{Study setting}

This study was conducted in the emergency department (ED) of King Abdulaziz Medical City (KAMC), which is a tertiary care hospital in Riyadh, the capital of Saudi Arabia. The ED at KAMC is composed of Urgi Center, Pediatric Emergency Units, Adult Emergency unit, Observation Unit, and Critical Care unit.

\section{Study design}

Retrospective cohort study of physician medication prescription over a period of 3 months from January to March 2008 in ED at KAMC, Riyadh, Saudi Arabia.

\section{Study population}

Charts of 101 patients diagnosed with UTIs due to E. coli were reviewed. Samples of those patients showed significant growth, bacteria growing $>10^{5}$ colony-forming units $(\mathrm{CFU} / \mathrm{mL})$ with a single type of bacteria from a properly collected midstream "clean catch" urine sample, were considered as UTIs and processed further for identification and susceptibility testing.

\section{Antibiotic sensitivity testing}

The sensitivity and resistance of $E$. coli isolates against six antimicrobial agents including ampicillin, augmentin, co-trimoxazole (trimethoprim/sulphamethoxazole), ciprofloxacin, nitrofurantoin, and cefazolin were determined using VITEK test method which measure minimum inhibitory concentration (MIC). Susceptibility results are interpreted according to the Clinical Laboratory Standards Institute (CLSI) guidelines. Then association between resistance of $E$. coli to one or more of three antibiotics (ampicillin, co-trimoxazole and ciprofloxacin) were studied and classified to SSR (sensitive to ampicillin, sensitive co-trimoxazole, and resistance ciprofloxacin); RRR (resistance ampicillin, resistance co-trimoxazole and resistance ciprofloxacin); SSS (sensitive ampicillin, sensitive co-trimoxazole and Sensitive ciprofloxacin); RRS (resistance ampicillin, resistance co-trimoxazole and sensitive ciprofloxacin); SRS (sensitive ampicillin, resistance co-trimoxazole and sensitive ciprofloxacin), and SRR (sensitive ampicillin, resistance co-trimoxazole and resistance ciprofloxacin). Multiple resistance which defined as multidrug resistance (MDR) was defined as resistance to three or more antimicrobials. The population was classified as pediatric, adolescent and adult, and elderly. Pediatric stratified into three groups: less than 2 years, from 2 years to 6 years and between 7 and 12 years. The age category between 13-17 and 18-64 years were defined as adults, while those age 65 years or over were defined as elderly.

\section{Measurement}

Data for the period of January 1, 2008, through March 30, were obtained from the prescription and Quadra Med system. All prescriptions with UTIs diagnosis were collected and reviewed for the demographic characteristic, the name of antibiotics and microbiology data. Microbiology data include name of the uropathogen which isolated from urine culture and cause UTIs, sensitivity, and resistance of $E$. coli for six antimicrobials agents as prescribed above.

\section{Statistical analysis}

Antimicrobial susceptibility or resistant rates were calculated as the number of susceptible or resistant organisms divided by the total number of tested organisms, for a given antibiotic, and a given organism. Statistical analyses were performed using the Statistical Package for the Social Sciences (SPSS version 16.0).

\section{Ethical approval}

This study approved by the research committee at King Abdullah International Medical Research Center, Riyadh, Saudi Arabia.

\section{Results}

During the study period from January to March 2008, a total 565 urinary cultures were collected. Of a total isolate, positive cultures were representing $28.67 \%$ of all cultures collected. Gram-negative organisms totaled 149 (91.98\%), Gram-positive organisms constituted 11 (6.79\%) and fungal 2 (1.23\%) of all isolates. There are $56.14 \%$ of all pediatric visits requested culture. $35.67 \%$ 
of all adult visits and $39.75 \%$ of all elderly. In pediatric, $32.29 \%$ of culture was positive, $62.50 \%$ was negative, and $5.21 \%$ of the result was mixed of normal flora, while in the adult the result of urine culture showed $68.62 \%$ negative, $24.34 \%$ positive and $7.05 \%$ was mixed. In elderly, the result of culture was $57.81 \%$ negative, $37.50 \%$ positive and $5.21 \%$ mixed of normal flora.

The most common uropathogen isolated from urine culture was E. coli, which represent $93.55 \%$ of all pathogen that isolated from pediatric urine culture, $60.24 \%$ of all pathogen which isolated from adult urine culture and $45.83 \%$ of all pathogen that isolated from elderly urine culture (Table 1). Extended Spectrum Beta-Lactamases enzymes (ESBL) producing E. coli detected in 8.33, 4.82 and $3.23 \%$ of uropathogens isolated from elderly, adult and pediatric respectively. Other organisms, in elderly, were Enterobacter species (10.42\%), Klebsiella species (10.42\%), and Acinetobacter species (6.25\%), and Pseudomonas aeruginosa (4.14\%). Other organisms were caused UTIs in adult were Klebsiella species (10.42\%) Pseudomonas aeruginosa (8.43\%), and Streptococcus species $(8.43 \%)$ (Table 1$)$.

The overall rate of resistance for $101 \mathrm{E}$. coli isolates analyzed was provided in Table 2 . Of the agents tested nitrofurantoin, ciprofloxacin and augmentin were the lowest rates of resistance in three age categories. Nitrofurantoin was the lowest rate of resistance among three age categories representing $(0,2,9 \%)$ followed by ciprofloxacin representing $(6.90,8,27.27 \%)$, and augmentin representing $(10.34,10,27.27 \%)$ in pediatric, adult and elderly

Table 1 Distribution of uropathogens that cause urinary tract infection according age category in period from January to March 2008

\begin{tabular}{|c|c|c|c|c|c|c|}
\hline \multirow[t]{3}{*}{ Uropathogens } & \multicolumn{6}{|c|}{ Frequency among patients } \\
\hline & \multicolumn{2}{|c|}{ Elderly $(n=48)$} & \multicolumn{2}{|c|}{ Adult $(n=83)$} & \multicolumn{2}{|c|}{ Pediatric $(n=31)$} \\
\hline & $\mathbf{n}$ & $\%$ & $\mathbf{n}$ & $\%$ & $\mathbf{n}$ & $\%$ \\
\hline Escherichia coli & 22 & 45.83 & 50 & 60.24 & 29 & 93.55 \\
\hline Enterobacter species & 5 & 10.42 & 4 & 4.82 & 0 & 0 \\
\hline Klebsiella species & 5 & 10.42 & 7 & 8.43 & 0 & 0 \\
\hline Extended spectrum B lactamase (ESBL) & 4 & 8.33 & 4 & 4.82 & 1 & 3.23 \\
\hline Acinetobacter species & 3 & 6.25 & 1 & 1.20 & 0 & 0 \\
\hline Group B sterptococcus & 2 & 4.17 & 7 & 8.43 & 0 & 0 \\
\hline Pseudomonas aeruginosa & 2 & 4.17 & 7 & 8.43 & 0 & 0 \\
\hline Candida species & 1 & 2.08 & 1 & 1.20 & 0 & 0 \\
\hline Morganella morganii & 1 & 2.08 & 0 & 0.00 & 0 & 0 \\
\hline Proteus merabilis & 1 & 2.08 & 1 & 1.20 & 1 & 3.23 \\
\hline Serratia marcescens & 1 & 2.08 & 0 & 0.00 & 0 & 0 \\
\hline Staphylococcus aureus & 1 & 2.08 & 0 & 0.00 & 0 & 0 \\
\hline Methicillin resistance Staphylococcus aureus (MRSA) & 0 & 0 & 1 & 1.20 & 0 & 0 \\
\hline
\end{tabular}

Table 2 Antimicrobial susceptibility result for $101 \mathrm{E}$. coli urinary tract isolates in study period from January to March 2008

\begin{tabular}{|c|c|c|c|c|c|c|}
\hline \multirow[t]{3}{*}{ Antibiotic name } & \multicolumn{6}{|c|}{ Number of isolates $\mathrm{n}(\%)$ classified as } \\
\hline & \multicolumn{2}{|c|}{ Pediatric $(n=29$ isolates) } & \multicolumn{2}{|c|}{ Adult ( $n=50$ isolates) } & \multicolumn{2}{|c|}{ Elderly ( $n=22$ isolates) } \\
\hline & Sensitive & Resistance & Sensitive & Resistance & Sensitive & Resistance \\
\hline Ampicillin & $5(17.24 \%)$ & $24(82.76 \%)$ & $21(42 \%)$ & $29(58 \%)$ & $8(36.36 \%)$ & $14(63.64 \%)$ \\
\hline Augmentin & $26(89.66 \%)$ & $3(10.34 \%)$ & $45(90 \%)$ & $5(10 \%)$ & $16(72.73 \%)$ & $6(27.27 \%)$ \\
\hline Co-trimoxazole & $14(48.28 \%)$ & $15(51.72 \%)$ & $29(58 \%)$ & $21(42 \%)$ & $9(40.91 \%)$ & $13(59.09 \%)$ \\
\hline Ciprofloxacin & $27(93.1 \%)$ & $1(3.44 \%)$ & $46(92 \%)$ & $4(8 \%)$ & $16(72.73 \%)$ & $6(27.27 \%)$ \\
\hline Nitrofurantoin & $29(100 \%)$ & $0(0.00 \%)$ & $49(98 \%)$ & $1(2 \%)$ & $20(90.91 \%)$ & $2(9.09 \%)$ \\
\hline Cefazolin & $25(86.21 \%)$ & $4(13.79 \%)$ & $43(86 \%)$ & $7(14 \%)$ & $15(68.18 \%)$ & $7(31.82 \%)$ \\
\hline
\end{tabular}

Co-trimoxazole (sulfamethoxazole/trimethoprim) 
respectively. Ampicillin demonstrated the highest rate of resistance $(82.76,58$, and $63.64 \%$ ) in pediatric, adult and elderly respectively. The rate of co-trimoxazole resistance among the $E$. coli isolates was $(51.72,42 \%)$, and $59.09 \%$ in pediatric, adult and elderly respectively (Table 2 ).

Antimicrobial resistance to individual antibiotics and the percentage of isolates demonstrating MDR phenotype were stratified by patient demographic characteristic and summarized in (Table 3). Nitrofurantoin resistance was approximately three times as common among E. coli isolates from males (7.14\%) more than females (2.3\%) and was highest $(9.09 \%)$ among patients $>65$ years old. Augmentin resistance was approximately comparable among E. coli isolates from male (14.29\%) and female (13.79\%) and the highest (27.27, and 15.78\%) among patients more than 65 years old and equal or less than 6 years old respectively. Higher rates of ciprofloxacin resistance (27.27\%) among patients > 65 years was demonstrated (Table 3). There was no resistance against augmentin and cefazolin among E. coli isolated from patients aged between 7 years to 17 years old. The rate of resistance of E. coli to co-trimoxazole was high in patients $<2$ years old and $>65$ years old representing $85.71 \%$ and $95.09 \%$, respectively. Trend toward higher rates of MDR E. coli were demonstrated among males' patients (28.57\%). By age category, the prevalence of MDR was higher in elderly $50 \%(\mathrm{n}=11$ of 22$)$, followed by adult $18 \%(\mathrm{n}=9$ of 50), and pediatric $10.34 \%(n=3$ of 29$)$ (Table 3$)$.

Among the $101 \mathrm{E}$. coli isolates that were tested against all six antimicrobials, $31.68 \%$ found to be susceptible (Table 4). The majority $(68.31 \%)$ was resistant to one or more antimicrobial and $15.84 \%$ were resistant to a single agent, predominantly ampicillin, and $29.7 \%$ were resistant to two antimicrobials. MDR isolates accounted for $22.77 \%(n=23)$ of the 101 isolates. The majority of MDR isolates $(\mathrm{n}=13 ; 56.52 \%)$ were resistant to three antimicrobial, and these accounted for $12.87 \%$ of all isolates. Isolates were also identified to be resistant to four agents were $(\mathrm{n}=8 ; 34,78 \%$ of MDR isolates; $7.92 \%$ of all isolates) and all five antimicrobials were $(\mathrm{n}=2 ; 8,69 \%$ of MDR isolates; $1.98 \%$ of all isolates) (Table 4). Resistant to ampicillin represent $34.84 \%(n=23$ of 23$)$ of the MDR isolates. Among 66 and 49 isolates resistant to ampicillin and co-trimoxazole, respectively, 34.84 and $42.85 \%$ were MDR (Table 4). In contrast, all isolates resistant to augmentin and nitrofurantoin were MRD, while 72.7 and $82.4 \%$ of isolates resistant to ciprofloxacin and cefazolin were MDR.

The analysis of co-resistance between ampicillin, co-trimoxazole and ciprofloxacin were summarized in Table 5 . The correlation between ampicillin and co-trimoxazole were $51.72,34$ and $27.27 \%$ in pediatric, adult and elderly respectively. $E$. coli were resistant to ampicillin, ciprofloxacin, and co-trimoxazole in adult and elderly were 4 and $27.27 \%$ respectively. In adult, the correlation between resistance of ciprofloxacin and co-trimoxazole was $2 \%$ (Table 5 ).

\section{Discussion}

Global spread of antibiotics resistance among uropathogens causing UTIs is alarming. This study reports the etiology of UTIs and antimicrobial susceptibility of uropathogens at KAMC in Riyadh, a capital city of Saudi Arabia. Similar to previous studies that conducted in Saudi Arabia, E. coli remains the majority of pathogen which isolated from urine culture in pediatric, adult, and elderly [11-14, 16], and other countries [17-19].

In this study, the resistance of E. coli to ampicillin was the highest, followed by co-trimoxazole. The observed resistance pattern of E. coli to ampicillin and co-trimoxazole are in agreement with the findings of previous studies carried out at different provinces of Saudi Arabia [11-13, 16]. Other study taken place in USA showed that that MDR E. coli exhibited 97.8\% resistance to Ampicillin, $92.8 \%$ to trimethoprim-sulfamethoxazole, and 38.8\% to ciprofloxacin [20]. In UK, high rates of resistance to ampicillin (55\%) and trimethoprim (40\%) were observed in E. coli isolates [18]. These results suggest the prevalence of ampicillin and trimethoprim/sulfamethoxazole resistance among urinary tract isolates and are consistent with finding of our study. E. coli showed the highest sensitivity to nitrofurantoin in the current study, which are in line with those of previous studies in Saudi Arabia $[12,14]$, and other countries [21, 22]. These data further support recommendations made in previous studies, in which, nitrofurantoin may be more effective than co-trimoxazole or amoxicillin in the empiric treatment of UTIs $[21,23]$.

The current data showed a high percentage of correlation between co-trimoxazole and ampicillin resistance among E. coli isolates (37.62\%). Previous data from Canadian study revealed limited activity of ampicillin $(79.6 \%$ resistance) against co-trimoxazole-resistant $E$. coli isolates from UTIs patients [24]. In the same study, the rates of co-trimoxazole resistance for ampicillin-resistant $E$. coli was (35.7\% resistance) [24], corroborating finding of our study. In addition, high ciprofloxacin resistant (100\%) was found among co-trimoxazole resistance isolates [24]. This finding is contrary to current study results as there is no evidence of high prevalence in ciprofloxacin resistant among ampicillin and/or co-trimoxazole resistant $E$. coli isolates from UTIs.

The current study found that $22.77 \%$ of $E$. coli isolates were MDR, which is higher than that observed in ED of previous study [25]. MDR prevalence was higher in E. coli isolates resistant to augmentin, nitrofurantoin, followed 


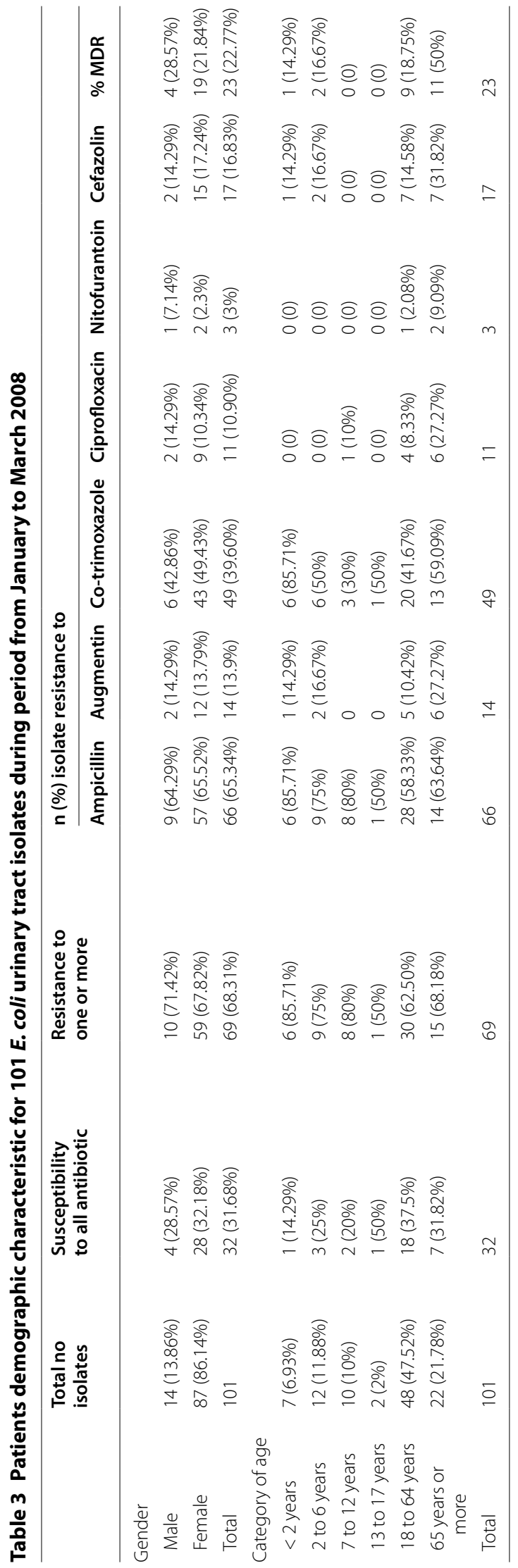


Table 4 Resistance to one or more antimicrobials among $101 \mathrm{E}$. coli urinary tract isolates tests against all six antimicrobials in study period

\begin{tabular}{|c|c|c|c|c|c|c|c|}
\hline \multirow{2}{*}{$\begin{array}{l}\text { No of agents which isolates were } \\
\text { resistance }\end{array}$} & \multirow[t]{2}{*}{$\mathrm{N}, \%$ of isolates } & \multicolumn{6}{|c|}{$\mathrm{n}(\%)$ isolates resistant to } \\
\hline & & Ampicillin & Augmentin & Co-trimoxazole & Ciprofloxacin & Nitrofurantoin & Cefazolin \\
\hline 0 & $32(31.68 \%)$ & & & & & & \\
\hline 1 & $16(15.84 \%)$ & $14(87.50 \%)$ & 0 & $2(12.50 \%)$ & 0 & 0 & $1(6.25 \%)$ \\
\hline 2 & $30(29.70 \%)$ & $29(96.67 \%)$ & 0 & $26(86.67 \%)$ & $3(10 \%)$ & 0 & $2(6.67 \%)$ \\
\hline 3 & $13(12.87 \%)$ & $13(100 \%)$ & $5(38.46 \%)$ & $12(92.31 \%)$ & $5(38.46 \%)$ & 0 & $5(38.46 \%)$ \\
\hline 4 & $8(7.92 \%)$ & $8(100 \%)$ & $7(87.5 \%)$ & $7(87.50 \%)$ & $2(25 \%)$ & $2(25 \%)$ & $7(87.50 \%)$ \\
\hline 5 & $2(1.98 \%)$ & $2(100 \%)$ & $2(100 \%)$ & $2(100 \%)$ & $1(50 \%)$ & $1(50 \%)$ & $2(100 \%)$ \\
\hline Total & 101 & 66 & 14 & 49 & 11 & 3 & 17 \\
\hline MDR & 23 & 23 & 14 & 21 & 8 & 3 & 14 \\
\hline$\% \mathrm{MDR}$ & $77.22 \%$ & $34.84 \%$ & $100 \%$ & $42.85 \%$ & $72.72 \%$ & $100 \%$ & $82.35 \%$ \\
\hline
\end{tabular}

Table 5 Resistance profile to the three antibiotics (ampicillin, ciprofloxacin and co-trimoxazole) among 101 isolates of $E$. coli

\begin{tabular}{llllllll}
\hline Antibiotype & Ampicillin & Co-trimoxazole & Ciprofloxacin & Pediatric n (\%) & Adult n (\%) & Elderly n (\%) & Total n (\%) \\
\hline A & S & S & S & $6(20.69 \%)$ & $19(38 \%)$ & $7(31.82 \%)$ & $32(31.68 \%)$ \\
B & R & R & S & $15(51.72 \%)$ & $17(34 \%)$ & $6(27.27 \%)$ & $38(37.62 \%)$ \\
C & R & R & R & 0 & $2(4 \%)$ & $6(27.27 \%)$ & $8(7.92 \%)$ \\
D & R & S & R & $1(3.45 \%)$ & $1(2 \%)$ & 0 & $2(1.98 \%)$ \\
E & R & S & S & $7(24.14 \%)$ & $9(18 \%)$ & $2(9.09 \%)$ & $18(17.82 \%)$ \\
F & S & R & R & 0 & $1(2)$ & 0 & $1(0.99 \%)$ \\
G & S & R & S & 0 & $1(2)$ & $1(4.55 \%)$ & $2(1.98 \%)$ \\
Total & & & & 29 & 50 & 22 & 101 \\
\hline
\end{tabular}

$S$ susceptible, $R$ resistance

by cefazolin and ciprofloxacin, then co-trimoxazole and ampicillin.

Despite the lowest rate of nitrofurantoin resistance (3\%) when compared to other antibiotics used in the current study (only 3 of 101), these isolates were MDR. Similarly, $100 \%$ of isolates resistant to augmentin were also MDR. However, in our study we described MDR phenotypes for ampicillin, co-trimoxazole, and ciprofloxacin but not for other antibiotics. Therefore, we cannot conclude the correction between MDR with specific antibiotic.

According to patient age, the prevalence of resistance to ampicillin or co-trimoxazole was higher among isolates from patients $\leq 12$ years and $\leq 2$ years, respectively. This pattern seemed relatively consistent with findings of previous study which revealed higher level of resistance among isolates from patients $\leq 17$ years old than among older patients [22]. For augmentin, cefazolin, ciprofloxacin, and nitrofurantoin, the prevalence of resistant isolates were higher among patients aged $\geq 65$ years. This match results observed in earlier studies that found ciprofloxacin resistance was highest among patients older than 65 years (7.1\%) [22]. However, nitrofurantoin resistance pattern in our study is contrary to that described by Sham et al. study, in which resistance prevalence of nitrofurantoin resistance was consistent irrespective of patient age [22].

The present study has some limitations. First, the study was only conducted only in ED setting, which not representing other health care settings in KAMC. Thus, the findings cannot be generalized. Second, the study period was short (3 months) and small sample size. Third, there was no follow-up study to investigate the consequences of observed resistance. Therefore, large-scale prospective studies are recommended to determine the extent and outcomes of resistance and MDR in UTIs at KAMC in Saudi Arabia.

\section{Conclusions}

Increased resistance of urinary tract $E$. coli isolates to both ampicillin and co-trimoxazole was demonstrated, suggesting revaluation empirical therapies for the treatment of UTIs in ED setting. This continuous evolvement in antimicrobial resistance pattern necessitates national 
surveillance studies to monitor and ensure safe and effective empiric therapy.

\author{
Abbreviations \\ KAMC: King Abdulaziz Medical City; KAIMRC: King Abdullah International \\ Medical Research Center, augmentin, (amoxicillin/clavulanate; ED: emergency \\ department, co-trimoxazole, (sulfamethoxazole/trimethoprim); UTIs: urinary \\ tract infections; MDR: multiple drug resistance; MRSA: methicillin resistance $S$. \\ aureus.
}

\section{Authors' contributions}

All authors contributed towards the study concept, data analysis, drafting, and revising of the paper and agreed to be accountable for all aspects of the work. All authors read and approved the final manuscript.

\section{Author details}

${ }^{1}$ Clinical Pharmacy Practice, Drug Policy and Economics Center, King Abdulaziz Medical City, Ministry of National Guard, Health Affairs, Riyadh 22490, Saudi Arabia. ${ }^{2}$ Department of PharmaceUTIscs, College of Pharmacy, King Saud University, Riyadh 22452, Saudi Arabia.

\section{Acknowledgements}

The authors would like to acknowledge the King Abdullah International Medical Research Center (KAIMRC) for the support. Also, authors would like to thank Prof. Amin Kashmeery, Dr. Abdullah Adlan, Prof. Abdulhaleem Sawas and Mohmmed Assiri from King Abdullah International Medical Research Center (KAIMRC). And special thank for Dr. Abdul Rahman Jazieh, Chairman Department of Oncology King Abdulaziz Medical City, Ministry of National Guard Health Affairs.

\section{Competing interests}

The authors declare that they have no competing interests.

\section{Availability of data and materials}

Data supporting the findings are included in the manuscript.

\section{Consent for publication}

Not applicable.

\section{Ethics approval and consent to participate}

This study approved by the research committee at King Abdullah International Medical Research Center, Riyadh, Saudi Arabia.

\section{Declarations}

This study was approved by the research committee at King Abdullah International Medical Research Center (RR08/005).

\section{Funding}

The study was supported by the Research Centre at King Abdullah International Medical Research Center (KAIMRC).

\section{Publisher's Note}

Springer Nature remains neutral with regard to jurisdictional claims in published maps and institutional affiliations.

Received: 31 December 2016 Accepted: 1 February 2018

Published online: 09 February 2018

\section{References}

1. Car J. Urinary tract infections in women: diagnosis and management in primary care. BMJ. 2006;332(7533):94-7.

2. Lee JBL, Neild GH. Urinary tract infection. Medicine. 2007:35(8):423-8.

3. Tandogdu Z, Wagenlehner FM. Global epidemiology of urinary tract infections. Curr Opin Infect Dis. 2016;29(1):73-9.
4. Taur Y, Smith MA. Adherence to the infectious diseases society of America guidelines in the treatment of uncomplicated urinary tract infection. Clin Infect Dis. 2007:44(6):769-74.

5. Schappert SM, Burt CW. Ambulatory care visits to physician offices, hospital outpatient departments, and emergency departments: United States, 2001-2002. Vital Health Stat. 2006;13(159):1-66.

6. Kostakioti M, Hultgren SJ, Hadjifrangiskou M. Molecular blueprint of uropathogenic Escherichia coli virulence provides clues toward the development of anti-virulence therapeUTIscs. Virulence. 2012;3(7):592-4.

7. Alanazi MQ, Al-Jeraisy MI, Salam M. Prevalence and predictors of antibiotic prescription errors in an emergency department, Central Saudi Arabia. Drug Healthc Patient Saf. 2015;7:103-11.

8. Gupta K, et al. The prevalence of antimicrobial resistance among uropathogens causing acute uncomplicated cystitis in young women. Int J Antimicrob Agents. 1999;11(3-4):305-8.

9. Kahlmeter G. The ECO.SENS Project: a prospective, multinational, multicentre epidemiological survey of the prevalence and antimicrobial susceptibility of urinary tract pathogens-interim report. J Antimicrob Chemother. 2000;46(Suppl 1):15-22 (discussion 63-5).

10. Foxman B. The epidemiology of urinary tract infection. Nat Rev Urol. 2010;7(12):653-60.

11. Al Yousef SA, et al. Clinical and laboratory profile of urinary tract infections associated with extended spectrum beta-lactamase producing Escherichia coli and Klebsiella pneumoniae. Ann Clin Lab Sci. 2016:46(4):393-400.

12. Kader AA, Kumar A. Prevalence and antimicrobial susceptibility of extended-spectrum beta-lactamase-producing Escherichia coli and Klebsiella pneumoniae in a general hospital. Ann Saudi Med. 2005;25(3):239-42.

13. Al-Harthi AA, Al-Fifi SH. Antibiotic resistance pattern and empirical therapy for urinary tract infections in children. Saudi Med J. 2008;29(6):854-8.

14. Al-Tawfiq JA, Anani AA. Antimicrobial susceptibility pattern of bacterial pathogens causing urinary tract infections in a Saudi Arabian hospital. Chemotherapy. 2009:55(2):127-31.

15. Foxman B, et al. Urinary tract infection: self-reported incidence and associated costs. Ann Epidemiol. 2000;10(8):509-15.

16. Al-Otaibi FE, Bukhari EE. Clinical and laboratory profiles of urinary tract infections caused by extended-spectrum beta-lactamase-producing Escherichia coli in a tertiary care center in central Saudi Arabia. Saudi Med J. 2013;34(2):171-6

17. Bours $\mathrm{PH}$, et al. Increasing resistance in community-acquired urinary tract infections in Latin America, five years after the implementation of national therapeUTIsc guidelines. Int J Infect Dis. 2010;14(9):e770-4.

18. Bean DC, Krahe D, Wareham DW. Antimicrobial resistance in community and nosocomial Escherichia coli urinary tract isolates, London 2005-2006. Ann Clin Microbiol Antimicrob. 2008;7:13.

19. Assefa A, et al. Bacterial profile and drug susceptibility pattern of urinary tract infection in pregnant women at Tikur Anbessa Specialized Hospital Addis Ababa, Ethiopia. Ethiop Med J. 2008;46(3):227-35.

20. Karlowsky JA, et al. Trends in antimicrobial resistance among urinary tract infection isolates of Escherichia coli from female outpatients in the United States. Antimicrob Agents Chemother. 2002:46(8):2540-5.

21. Gupta K, Scholes D, Stamm WE. Increasing prevalence of antimicrobial resistance among uropathogens causing acute uncomplicated cystitis in women. JAMA. 1999;281(8):736-8

22. Sahm DF, et al. Multidrug-resistant urinary tract isolates of Escherichia coli: prevalence and patient demographics in the United States in 2000 Antimicrob Agents Chemother. 2001:45(5):1402-6.

23. Cormican $M$, et al. Extended spectrum beta-lactamase production and fluorquinolone resistance in pathogens associated with community acquired urinary tract infection. Diagn Microbiol Infect Dis. 1998;32(4):317-9.

24. Zhanel GG, et al. A Canadian national surveillance study of urinary tract isolates from outpatients: comparison of the activities of trimethoprimsulfamethoxazole, ampicillin, mecillinam, nitrofurantoin, and ciprofloxacin. The Canadian Urinary Isolate Study Group. Antimicrob Agents Chemother. 2000:44(4):1089-92.

25. Khawcharoenporn T, Vasoo S, Singh K. Urinary tract infections due to multidrug-resistant enterobacteriaceae: prevalence and risk factors in a Chicago Emergency Department. Emerg Med Int. 2013;2013:258517. 